This item was submitted to Loughborough's Research Repository by the author.

Items in Figshare are protected by copyright, with all rights reserved, unless otherwise indicated.

\title{
Monitoring dynamic structural tests using image deblurring techniques
}

PLEASE CITE THE PUBLISHED VERSION

http://www.ttp.net/978-3-03785-796-0.html

PUBLISHER

(c) Trans Tech Publications Ltd

VERSION

AM (Accepted Manuscript)

LICENCE

CC BY-NC-ND 4.0

REPOSITORY RECORD

McCarthy, David M.J., Jim H. Chandler, and Alessandro Palmeri. 2019. "Monitoring Dynamic Structural Tests Using Image Deblurring Techniques". figshare. https://hdl.handle.net/2134/12804. 
This item was submitted to Loughborough's Institutional Repository (https://dspace.lboro.ac.uk/) by the author and is made available under the following Creative Commons Licence conditions.

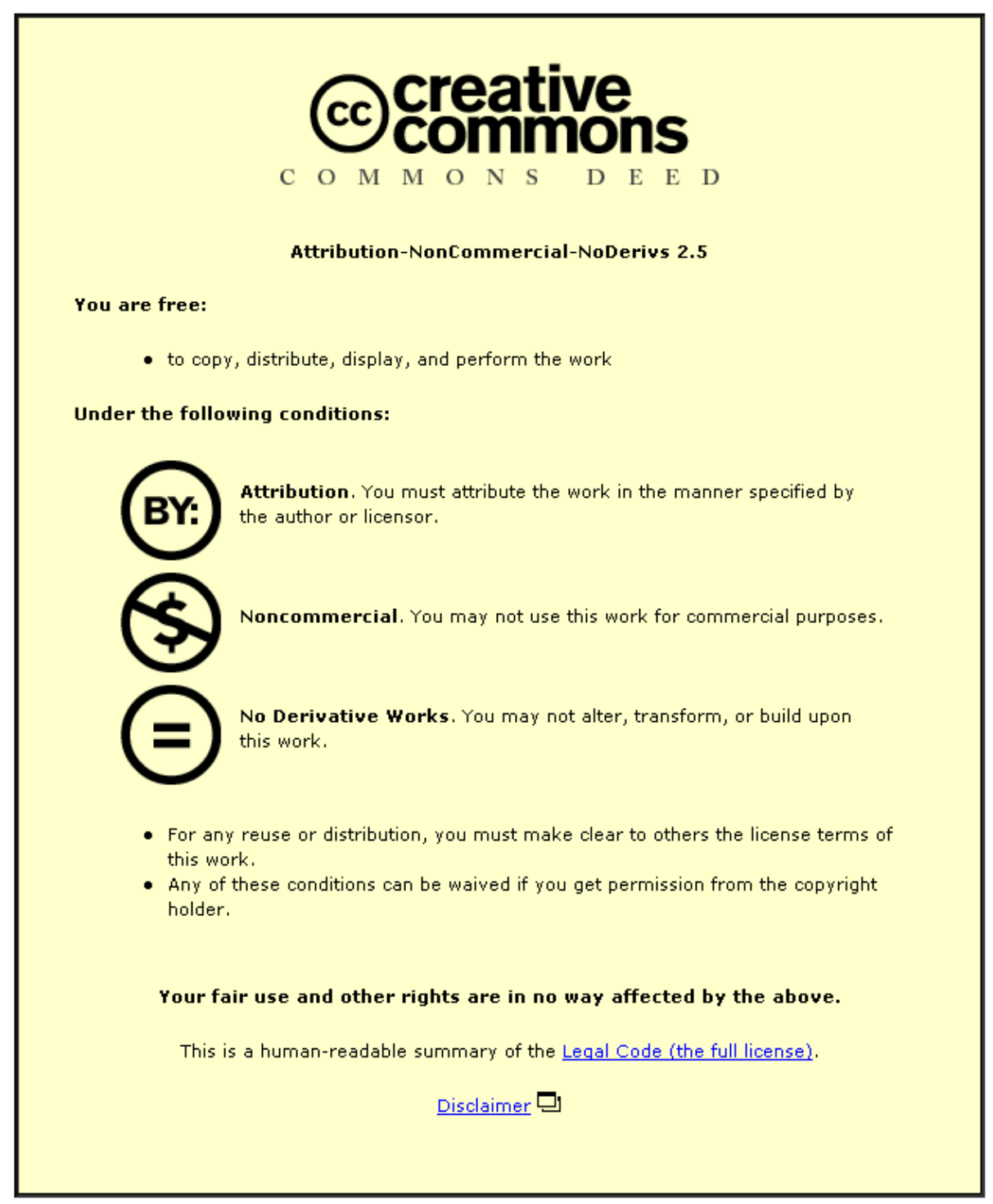

For the full text of this licence, please go to: http://creativecommons.org/licenses/by-nc-nd/2.5/ 


\title{
Monitoring Dynamic Structural Tests using Image Deblurring Techniques
}

\author{
D.M.J.McCarthy ${ }^{1, a}$, J.H.Chandler ${ }^{1, b}$ and A.Palmeri ${ }^{1, c}$ \\ ${ }^{1}$ School of Civil and Building Engineering, Loughborough University, \\ Loughborough, Leics, LE11 3TU \\ a'D.McCarthy@lboro.ac.uk, b.H.Chandler@lboro.ac.uk, '․P.Palmeri@lboro.ac.uk
}

Keywords: Dynamic testing, Image processing, Structural health monitoring, Image deblurring, Point spread function, Close range photogrammetry, Vibration measurement.

\begin{abstract}
Photogrammetric techniques have demonstrated their suitability for monitoring static structural tests. Advantages include scalability, reduced cost, and three dimensional monitoring of very high numbers of points without direct contact with the test element. Commercial measuring instruments now exist which use this approach.

Dynamic testing is becoming a convenient approach for long-term structural health monitoring. If image based methods could be applied to the dynamic case, then the above advantages could prove beneficial. Past work has been successful where the vibration has either large amplitude or low frequency, as even specialist imaging sensors are limited by an inherent compromise between image resolution and imaging frequency. Judgement in sensor selection is therefore critical. Monitoring of structures in real-time is possible only at a reduced resolution, and although imaging and computer processing hardware continuously improves, so the accuracy demands of researchers and engineers increase.

A new approach to measuring the vibration envelope is introduced here, whereby a long-exposure photograph is used to capture a blurred image of the vibrating structure. The high resolution blurred image showing the whole vibration interval is measured with no need for high-speed imaging. Results are presented for a series of small-scale laboratory models, as well as a larger scale test, which demonstrate the flexibility of the proposed technique. Different image processing strategies are presented and compared, as well as the effects of exposure, aperture and sensitivity selection. Image processing time appears much faster, increasing suitability for real-time monitoring.
\end{abstract}

\section{Introduction}

Image-based monitoring using photogrammetric techniques has been demonstrated for structural testing. The approach allows non-contact monitoring of a high number of points with one (for 2D monitoring) or two or more (for 3D deformations) imaging sensors. The approach is scalable, and for the amount of data collected, relatively inexpensive.

The approach has been demonstrated for monitoring dynamic structural tests, but monitoring is limited by a compromise between sensor resolution and sampling frequency. Real-time monitoring is possible but only at a reduced resolution because of the huge amount of data produced by high speed imaging sensors. To improve measurement accuracy, telephoto lenses have been used to 'zoom-in' on a single target [1], but the advantage of many monitoring points is lost.

A new approach is introduced in this paper where a long-exposure image is used to capture the whole vibration. The vibration interval is recorded within the single image and an algorithm for its measurement demonstrated. The camera sensor can be of higher resolution than those previously used as there is no requirement for high speed imaging, allowing more accurate spatial measurement.

\section{Structural Testing}

Structural testing is used to evaluate the performance of structural members, both of in situ structures and in the laboratory environment. Structural testing may be carried out to test correlation 
with analytical models of structural behaviour. Typically, loads are applied to test structural elements, and their performance monitored as the load increases to the target limit. Some tests may demand more advanced monitoring systems to record complex deformation patterns

The integrity of existing structures may also wish to be tested. The structure may be overloaded above its intended working load and based on its performance, deemed safe for its intended use. The record of the test serves as evidence of the structure's integrity. A structure may also be tested if, for example, a change of use is desired, to verify its integrity following a potentially damaging event, or if the quality of repair work needs to be verified. Structural health monitoring programs for monitoring the long-term status of structures are becoming more common.

Dynamic structural testing. Dynamic testing techniques, whereby the structural vibration is recorded, are a prevailing approach for structural health monitoring. The testing approach can be considered convenient for long-term monitoring, as light structural changes can be detected without the associated inconvenience of static testing. Interpreting the vibration response data could be, however, more complicated and specialised expertise is required to quantify damage.

Typically, changes in natural frequencies would be detected, which would indicate some change in stiffness. The more advanced algorithms use discontinuities in mode shapes to predict location and estimate severity [2]. Structural mode shapes are found by monitoring at several locations on a structure. For these methods, a higher number of sensors provides the most accurate results [3,4].

Accelerometers, commonly used for structural testing, are relatively inexpensive and simple to use. However, installing a number of them on to a structure becomes expensive, with associated cabling infrastructure for power and data and/or additional wireless equipment. Data from standard accelerometers may also not measure very low frequency vibrations [5].

\section{Imagery-based monitoring of structures}

Image-based monitoring has proven its application in the past to monitoring structural tests [5-8]. A structure is measured using photogrammetric techniques and data compared at different time intervals to calculate displacements. Advantages include: measurement can be made without contact with the structure, making it suitable for difficult measuring tasks where there is no stable support for displacement gauges. A very high number of points can be measured by a single camera sensor, limited only by sensor resolution, allowing monitoring of complex deformation patterns. Multiple camera sensors can monitor deformation in three dimensions.

While the most accurate measurement work still requires targets to be fixed to structures, digital image correlation (DIC) makes possible measurement of structures without targets fitted, provided their surface has sufficient natural texture [5]. Not needing to measure individually fitted targets allows very densely distributed measuring points and 'full-field' measurement. This allows visualisation of, for example, strain gradients, without long preparation and time on-site.

The scalability of image processing means measurements of large structures are possible to submillimetre accuracy while the accuracy of measuring small scale laboratory tests can be in the order of microns. Demonstrations give laboratory-scale examples of monitoring complex deformation in beam load tests as well as measuring the width of small scale hairline cracks in concrete [6-8]. Mass and Hampel [8] also give examples of large scale monitoring of complex structures such as buildings, bridges and reservoir dams.

GPS has also been proposed as an approach for monitoring static and dynamic structural testing [9]. Although requiring a base station receiver near the structure in addition to the receiver on the structure, line-of-sight between the two is not required. The instruments necessary for a usable accuracy are expensive and specialised and additional monitoring points radically increase costs.

\section{Proposed Vibration Measurement Methodology}

The advantages of photogrammetric image processing for monitoring structural testing can be taken advantage of in the dynamic case also. In dynamic testing, the principle remains the same, but the rate of image acquisition is increased to many pictures per second. This has been demonstrated 
[10-12] but current hardware is limited by a compromise between sensor resolution and the number of frames per second that can be recorded. The choice of sensor is important, as the number of frames per second will limit the frequency of vibrations that can be recorded and an inadequate sensor resolution will fail to detect low amplitude vibration. The Nyquist sampling theorem states that in order to measure a vibration, the sampling frequency must be at least two times the vibration frequency. Very high resolution sensors can measure many targets over a whole structure, but need to record at below $10 \mathrm{~Hz}$. Other sensors can record at up to $1000 \mathrm{kHz}$, but can only measure a very limited number of targets within close proximity.

The proposed approach. Our approach involves capturing a long-exposure blurred image of circular targets marked on the structure. High-speed imaging is not necessary as a 'smear' of the whole blur interval is captured in the single image. The vibration amplitude at a high number of points on the structure is measured and this information can be subsequently related to the expected performance of the structural system under the test load. Our implementation involves capturing a sharp image through a fast exposure as well as the blurred, which are processed in combination.

Measuring blur in computer science. A keen research area in computer science is that of 'image deblurring'. Algorithms exist that take a single blurred image and impressively 'de-blur' it. Rather than simply sharpening edges in images, these de-blurring algorithms first estimate the motion that caused the blur before applying a correction based on the estimated blur shape and size [13]. Artificial markings are not always required, but there is dependence on sufficient object texture.

The first stage of estimating the motion causing the blur is key to a successful 'deblur', and there are many advanced algorithms published. After estimating motion, other algorithms exist that estimate the spatial movement such as the speed of moving balls in sports [14]. The size and shape of the estimated motion is conveniently described by the point spread function (PSF) and measuring the estimated PSF provides the extent of motion at that point in an image. The PSF also contains data about the amount of time the moving object spent in each position. This approach, while alleviating the need for object targets, is computationally intensive, requiring several minutes to iteratively estimate the motion at a single point; this long processing time precludes real-time monitoring. Incorrect results are also common where surface texture is poor, yet it is difficult to identify these incorrect motion estimations.

Matlab Implementation. Our Matlab implementation processes images containing circular targets in three stages with increasing accuracy (but decreasing speed).

First: Estimate with threshold. First, a threshold filter is used to quickly identify the centre of the blurred target and estimate of the size and shape of the blur (Fig. 1a). This step also determines

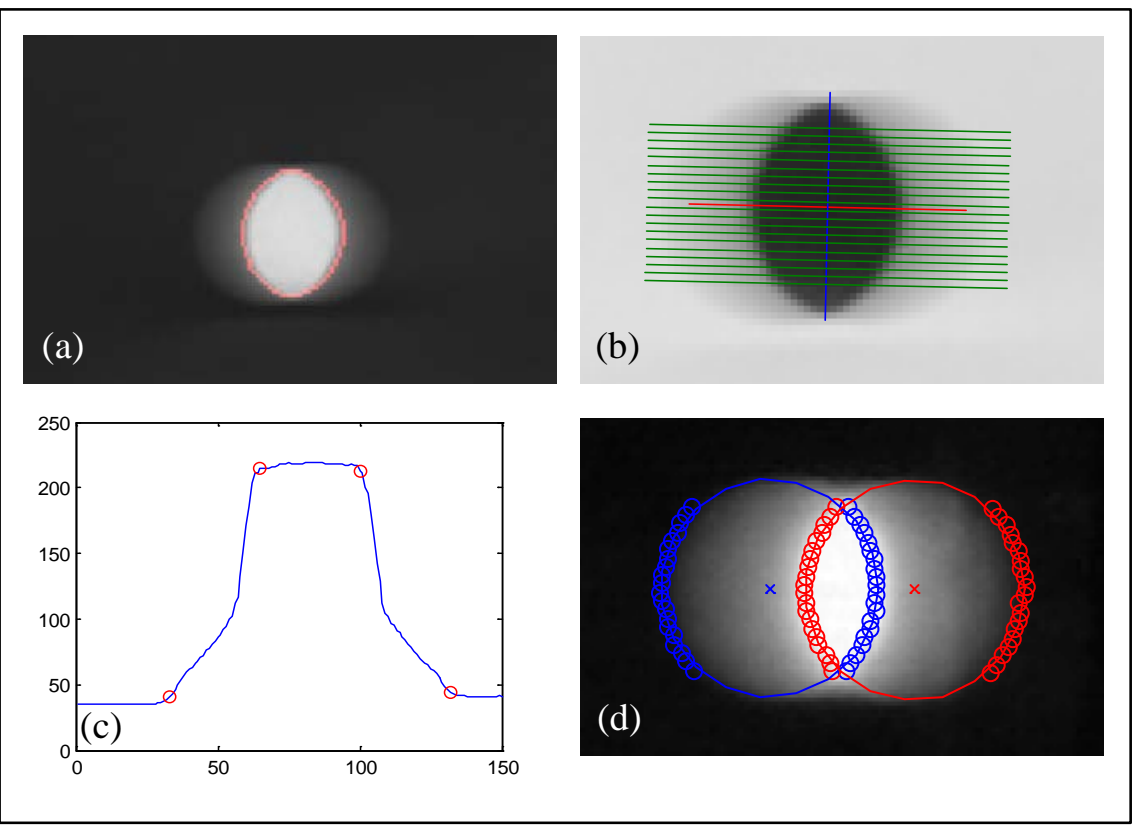

Figure 1: Estimate for blur dimensions using pixel intensity profiles. (a) First shape estimate, (b) Produced profile lines, (c) Individual intensity profile with identified blur edges, (d) Measured blur dimensions. 
whether the blur is an 'internal' blur, where the vibration amplitude is less than the diameter of the circular target, or an 'external' blur, where the vibration amplitude is greater. In the first case, part of the image is always occupied by the circular target and a patch of low intensity pixels will exist, in the second case there is a smaller range of pixel values. For internal blurs, an ellipse fit to this low intensity patch will have a major axis roughly perpendicular to the blur orientation (Fig. 1a).

Second: Pixel intensity profiles. We then use the approach proposed by Boracchi et al. [15] for estimating the motion of sports balls. The algorithm is initialised by using a simple threshold filter with a higher threshold to better estimate the size and orientation of the blur (Fig. 1b). A number of intensity profiles are made within the blur. Four points of interest are picked from the profile: at the start and end of the positive gradient, and the start and end of the negative gradient (Fig. 1c). The algorithm that picks these points is the same for both internal and external blur cases. An ellipse is fit to each set of points for each edge of the blur and the difference between the centres of the two ellipses identifies the amplitude of the vibration.

Although the shape of intensity profiles are consistent, the current implementation is susceptible to some image noise, resulting in some outliers. A quality check is important so that these outliers can be detected and corrected or removed.

Third: Simulated blur smears. To improve upon the previous analysis, the third proposed stage reverses deblurring algorithms by iteratively simulating different blur dimensions of the sharp image and correlating with the observed blurred image (Fig. 2). This builds on the assumption that a blurred image can be represented as an un-blurred image filtered by some motion filter. This provides subpixel (and depending on scale, sub-millimetre) accuracy as well as a measure of quality.

\section{Initial Experiments}

To test the algorithm, simulations were carried out on a small $(30 \times 30 \mathrm{~cm})$ shake table at Loughborough University (Fig. 3). Small plastic models were built, and targets adhered on to them. A single Nikon D80 DSLR camera was set up on a tripod to monitor the model. The vibration of the structure was measured by accelerometers, and the table bed position was also monitored by a laser sensor.

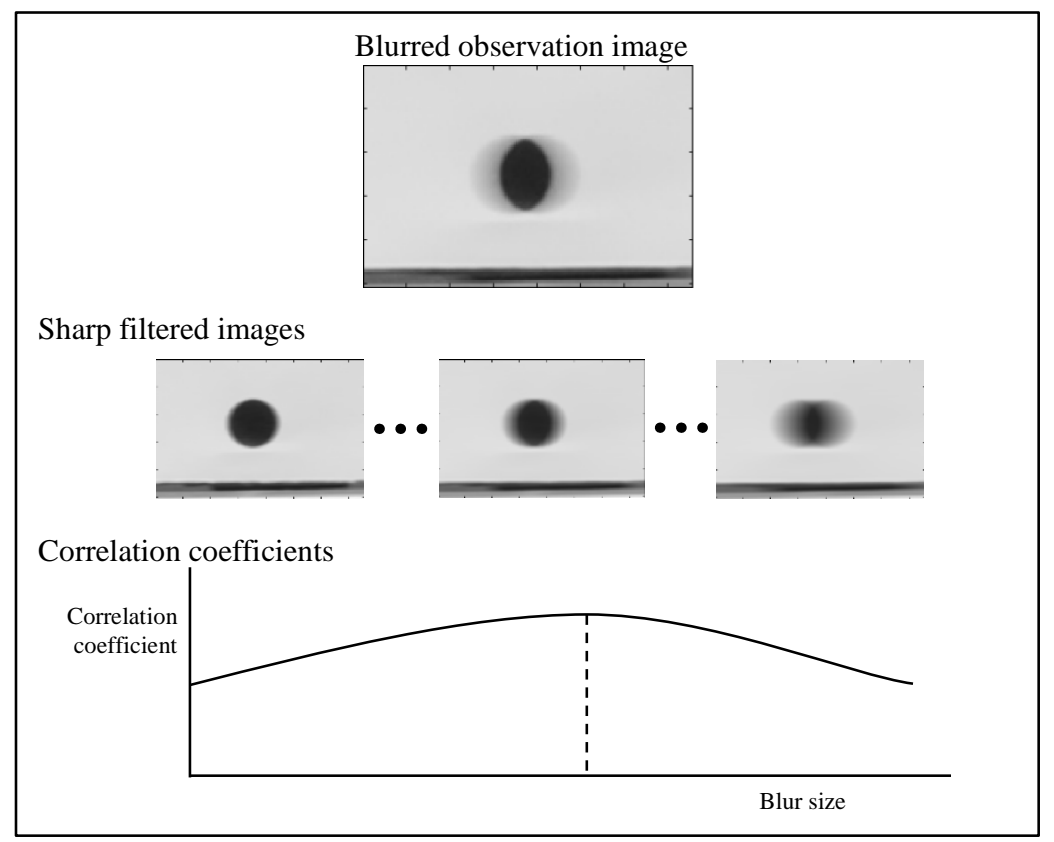

Figure 2: Blur measurement by simulation for sub-pixel precision and validation 


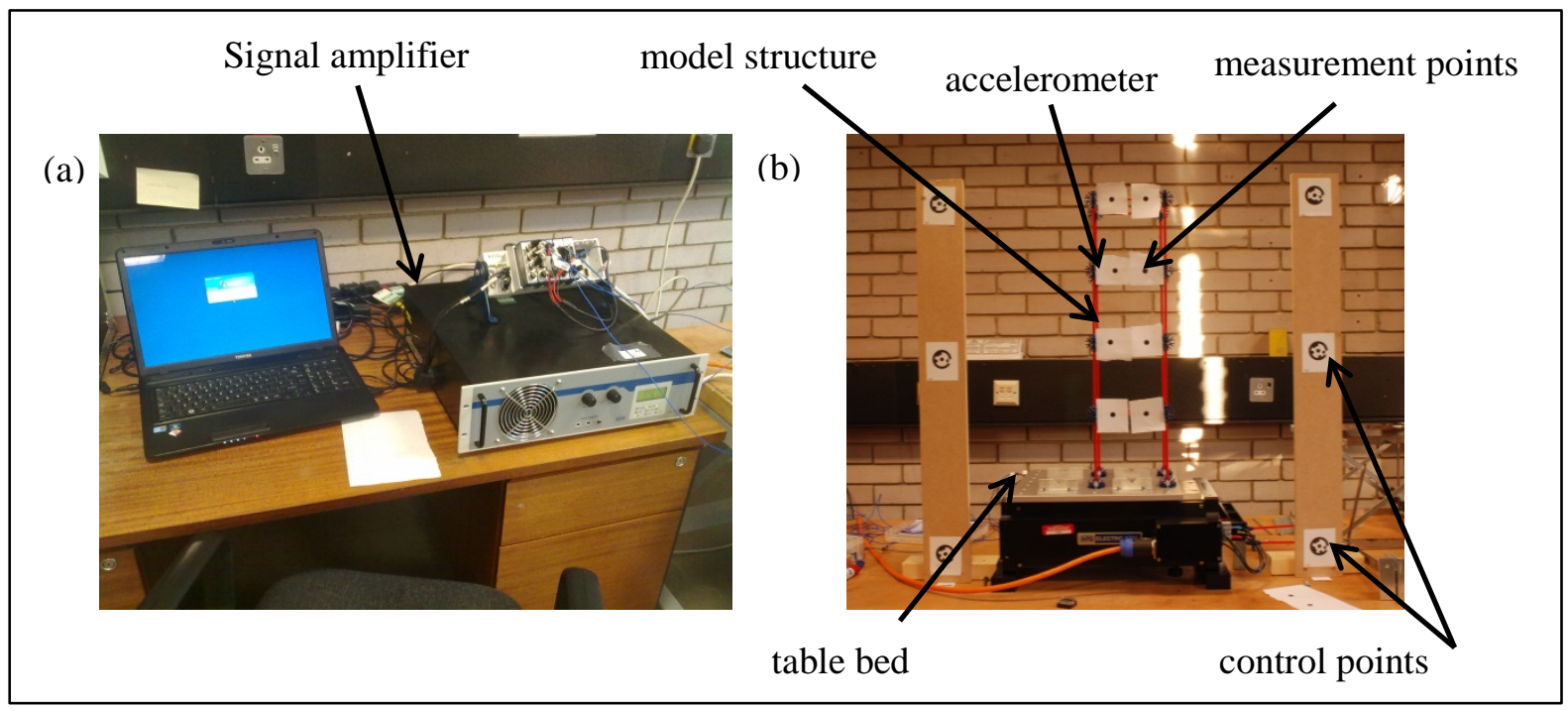

Figure 3: Shake table experiments at Loughborough University (a) table control equipment (b) sample image

The two wooden stands either side of the shake table support control points, stationary targets used to provide scale. In this instance, only planar movements were recorded, as all targets lay in a single plane. Displacements measured in the image space were transformed to real word coordinates in millimetres using the projective transformation. The control targets were measured using a Leica TCR400 reflectorless total station.

To assess the accuracy of the measured blurs, the shake table was programmed to vibrate with simple harmonic motion of varying amplitudes. Using the displacement laser and double integrating the accelerometer data, the vibration amplitude measured by the image processing approach is compared in Fig 4. Accuracies of $0.25 \mathrm{~mm}$ are achieved (using a 95\% confidence interval), which is further discussed in the following section. It is worth noting that we are interested in the envelope of the dynamic response of the sample structure, independently of the frequency of vibration.

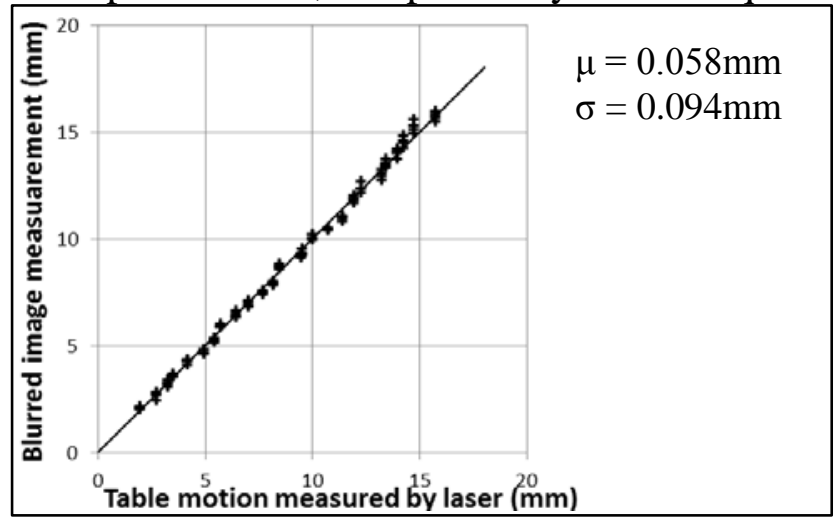

Figure 4: Results (at present scale, $1 \mathrm{~mm} \approx 3$ pixels)

\section{Discussion}

The spatial accuracy of photogrammetric measurements is dependent upon the scale of the test, so accuracy in pixels can be used to compare to other photogrammetric work. At the scale used, an accuracy of $0.25 \mathrm{~mm}$ (using a 95\% confidence interval) is about 0.64 pixels in the image space which is unimpressive when compared with that of traditional photogrammetry using sharp images [16], but because recording dynamic tests with high-speed cameras necessitates lower image resolutions, even with specialist imaging sensors, the actual real-space measurement accuracy is higher than that previous achieved. It was noted that the accuracy of the largest vibration amplitudes had the highest measurement error, probably due to low range of pixel intensity values in the fainter image and lower signal-to-noise ratio.

The output is also frequency-independent, as the measureable vibration is not dictated by the Nyquist criterion, and aliasing, whereby a peak of vibration is missed, does not occur. 
Demonstrations here use only a few targets, but the number of possible targets is limited only by practical considerations of attaching them to the structure, allowing many more. Increasing the number of targets would allow more accurate measurement of mode shapes with less interpolation. Increasing targets further, especially in a laboratory environment, approaches full-field measurement. If accelerometers or GPS were used, additional hardware would be necessary for each monitoring point, and this solution is much less expensive that the multiple-point-monitoring scanning laser Doppler vibrometer alternative.

Low frequency vibrations $(<1 \mathrm{~Hz})$ are measured in the same way by using a longer shutter speed. Shutter speeds of several seconds cause images to become overexposed, but this is mitigated with lens filters. The same setup is also suitable for monitoring of static properties of the bridge using well-known photogrammetric techniques. These properties when measured with an accelerometer are susceptible to inaccuracies due to 'drift' in the accelerometer data [1].

Practical considerations. The imaging hardware used is a consumer grade DSLR camera, and processing is carried out with an average specification PC. Such an approach allows a flexible monitoring scheme using off-the-shelf components. The current setup requires fixing targets in visible positions on the structure and control targets around the outside, which are then measured by total station. However, a simple scale constraint could be the only control required, provided by a simple scale ruler. Time to set up is comparable to other non-contact optical monitoring techniques which do not require the cabling infrastructure of many individual sensors.

The approach is, however, limited in its requirement of a suitable imaging station. The fixed control is also necessary to monitor for any camera movement in between measurement epochs. The requirement for control on a stable reference may be restrictive in outdoor applications, as a stable reference near tall structures may not be available. The targets demonstrated here would only be visible during daylight hours, although 'active' targets containing LEDs have been proposed and evaluated by others [1].

Image processing considerations. Processing time is a matter of seconds on an average specification computer. While not producing immediate real-time results to the user, it does produce results rapidly.

The current implementation requires the user must manually specify the initial region of interest and a small number of parameters. After this, processing of a number of similar images is automated, even where there is a high number of measuring points within the image. It is anticipated that the few manual parameters which relate to, for example, the lighting and colour of target against the background, could also be set automatically.

Camera exposure and response function. Particular care has to be given to selecting the correct exposure setting of the camera. Saturated areas of the image, whereby some pixels are overexposed, are not unlikely to occur when using long exposure images, and the overexposed area contains no gradient information. The solution is to reduce the exposure, but this can only be realised after the first image has been taken. In the anticipated real-time processing solution, saturation will be automatically realised after the first image is taken and the exposure time can be quickly corrected.

Borracchi et al. [15] suggested that most cameras do not apply a linear relationship between the amount of light at each pixel on the sensor and final pixel value recorded. This was particularly apparent when comparing the blurred observation image with the sharp filtered image. Although the two images were very similar (Fig. 2), containing similar edges, gradients and artefacts, the overall intensity of the 'smear' differed. Borracchi et al. [15] suggest that modelling the camera's transfer function for pixel intensity values will correct for this.

The blur distance measured by pixel intensity profiles, as was proposed by Boracchii et al. [15], regularly overestimates the actual vibration interval. This is because, although most of the gradient from target to background is caused by the motion 'smear', some gradient with a width of about 1-2 pixels is normally present around ordinary images of photogrammetric targets. This is evident when using the same algorithm on stationary targets, were movement is estimated of about the width of the target fringe. This systematic error may have been insignificant in Boracchii et al.'s 


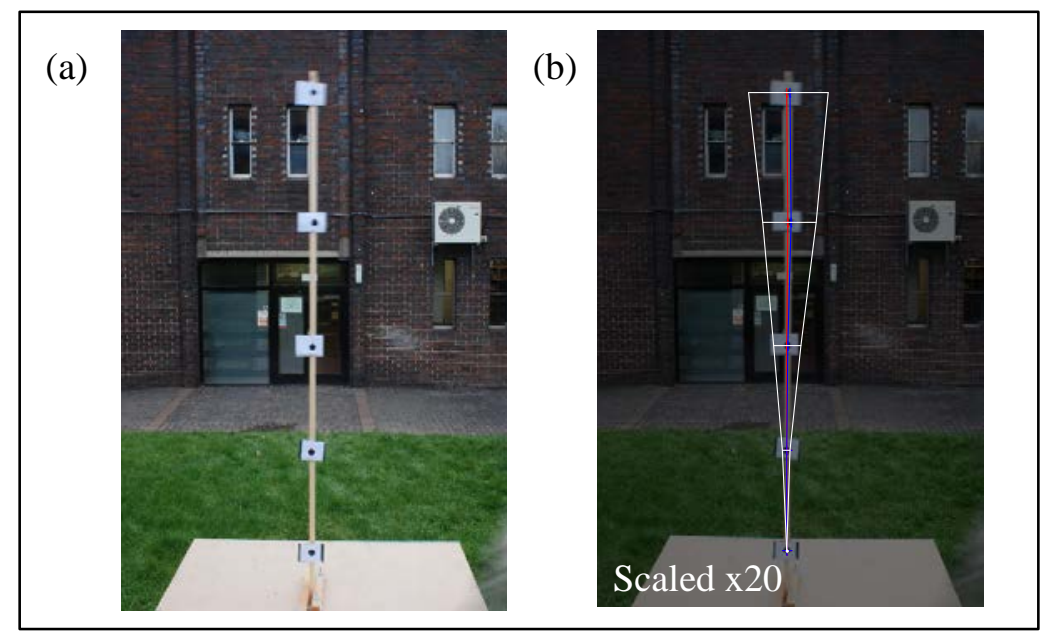

Figure 5: Demonstration capturing a motion-blurred image outdoors

(a) the tested column (b) the measured vibration envelope (scaled x20)

work which used images of a different scale, but for our work it should be anticipated and corrected. In our solution, the iterative simulation method used for sub-pixel measurement has the further advantage that it does not contain this systematic error.

\section{Future applications}

The scalability of the approach allows image processing to be carried out by the same algorithm on small as well as larger-scale structures. The approach will now be tested on larger full-scale structures.

Feasibility test for larger structures. The accuracy for small-scale tests shown above was approximately $0.2 \mathrm{~mm}$ for a $0.5 \mathrm{~m}$ tall model observed at about $2 \mathrm{~m}$. In the image space, this is an accuracy of 0.6 pixels. A larger structure, for example with a height of $5 \mathrm{~m}$, could be observed with the same lens hardware and an accuracy of less than a millimetre would be expected.

A likely additional challenge would be the lack of control over lighting conditions, although it is anticipated that adjusting camera exposure settings will account for this. Sample images of simple outdoor structures (Fig. 5) indicate that image processing is successful, with careful selection of exposure settings.

In comparison to GPS monitoring of structures, the measurement of vibration amplitude would be expected to be more accurate. Like photogrammetry, GPS is also suitable for static and semistatic monitoring approaches, but it is only sensitive to vibration with amplitudes above about 5-10 mm. While GPS data does contain frequency information, the proposed image processing approach would allow measurement of structures with lower vibration amplitudes.

Estimating the frequency of the dominant mode. Current exposure times for the current demonstrations have been large enough to capture the whole vibration interval. If the exposure time is reduced, and a structure's vibration remains constant, the image will record only part of the structure's vibration. It is suggested that there is potential for estimating the frequency of a dominant mode of a structure by measuring how much of a structure's whole vibration interval is occupied within the time the shutter is open.

\section{Conclusion}

An approach to estimating the vibration envelope using long-exposure single images has been proposed. Previous image monitoring systems have been limited by a compromise between spatial resolution and imaging frequency, even when using specialist sensors. This frequency-independent approach avoids this limitation and has been demonstrated using consumer-grade imaging hardware. An algorithm for correlating the observed image with simulated images has demonstrated the efficacy of the approach.

When used with frequency-selective narrowband excitation, the approach provides spatial measurement of vibration amplitudes more accurately than previous high-speed imaging solutions. The high number of possible measurement points with image-based techniques is highlighted as of potential benefit to determining mode shapes as it reduces the distance between measurement points that otherwise requires interpolation. 
Consideration should be given to the relatively low cost of the instruments used and the scalability of the approach. Because measurement is frequency-independent, hardware is not limited to certain frequency ranges. Initial tests on small-scale models have shown the approach can measure with $0.25 \mathrm{~mm}$ accuracy, which is scalable to structures of other sizes. The feasibility of monitoring larger full-scale structures has been tested and accuracy estimated. Finally, further work to assess the potential for frequency measurement has been introduced.

\section{References}

[1] Wahbeh, A.M., Caffrey, J.P. \& Masri, S.F., A vision-based approach for the direct measurement of displacements in vibrating systems. Smart materials and structures. 12 (2003) 785-794.

[2] Pandey, A., Biswas, M. \& Samman, M., Damage detection from changes in curvature mode shapes. Journal of Sound and Vibration. 145(2) (1991) 321-332.

[3] Carden, E.P., Vibration Based Condition Monitoring: A Review. Structural Health Monitoring. 3(4) (2004) 355-377.

[4] Dilena, M. \& Morassi, A., Dynamic testing of a damaged bridge. Mechanical Systems and Signal Processing. 25(5) (2011) 1485-1507.

[5] Warren, C., Niezrecki, C., Avitabile, P. \& Pingle, P., Comparison of FRF measurements and mode shapes determined using optically image based, laser, and accelerometer measurements. Mechanical Systems and Signal Processing. 25(6) (2011) 2191-2202.

[6] Robins, P., Austin, S., Chandler, J.H. \& Jones, P., Flexural strain and crack width measurement of steel-fibre-reinforced concrete by optical grid and electrical gauge methods. Cement and Concrete Research. 31(5) (2001) 719-729.

[7] Lange, J. \& Benning, W., Crack detection at concrete construction units from photogrammetric data using image processing procedures, in: N. Kerle \& A. Skidmore, (Eds.), Proceedings of the ISPRS Commission VII Symposium “Remote Sensing: From Pixels to Processes”, Enschede, The Netherlands, 2006.

[8] Maas, H. \& Hampel, U., Photogrammetric Techniques in Civil Engineering Material Testing and Structure Monitoring. Photogrammetric Engineering \& Remote Sensing. 72(1) (2006) 39-45.

[9] Roberts, G.W., Meng, X., Brown, C. J. \& Dallard, P. GPS measurements on the London Millennium Bridge. Proceedings of the ICE - Bridge Engineering. 159(4) (2006) 153-161.

[10]Lee, J.-J. \& Shinozuka, M., Real-Time Displacement Measurement of a Flexible Bridge Using Digital Image Processing Techniques. Experimental Mechanics. 46(1) (2006) 105-114.

[11]Park, J.-W., Lee, J.-J., Jung, H.-J. \& Myung, H., Vision-based displacement measurement method for high-rise building structures using partitioning approach. NDT \& E International. 43(7) (2010) 642-647.

[12]Choi, H.-S. Cheung, J.-H., Kim, S.-H., \& Ahn, J.-H., Structural Dynamic Displacement Vision System using Digital Image Processing. NDT \& E International. 44(7) (2011) 597-608.

[13]Fergus, R., Singh, B. \& Hertzmann, A., Removing camera shake from a single photograph. ACM Transactions on Graphics. 1(212) (2006) 787-794.

[14]Caglioti, V. \& Giusti, A., Recovering ball motion from a single motion-blurred image. Computer Vision and Image Understanding. 113(5) (2009) 590-597.

[15]Boracchi, G., Caglioti, V. \& Giusti, A., Ball position and motion reconstruction from blur in a single perspective image, in: Image Analysis and Processing. Modena: IEEE Comput. Soc., 2007.

[16]Luhmann, T., 3D Imaging - How to Achieve Highest Accuracy, in: F. Remondino \& M. R. Shortis, (Eds.), Proc. of SPIE: Videometrics, Range Imaging, and Applications XI, Munich, Germany, 2011, pp. 808502-1-11. 